\title{
Cyclin D1 Gene Numerical Imbalances in Laryngeal Squamous Cell Carcinoma: A Tissue Microarray Grid Based Analysis
}

\author{
Efthymios Kyrodimos ${ }^{1}$, Vasileios Papanikolaou ${ }^{1}$, Evangelos Tsiambas ${ }^{2,3 *}$, \\ Dimitrios Kikidis $^{1}$, Dimitrios Peschos ${ }^{4}$, Vasileios Ragos ${ }^{5}$, Nicholas Mastronikolis ${ }^{6}$, \\ Christos Riziotis $^{7}$, Aristeidis Chrysovergis ${ }^{1}$
}

\begin{abstract}
Background: Deregulation of critical proteins involved in cell cycle stability, such as cyclins, is a frequent genetic event in the development and progression of solid malignancies. Concerning laryngeal squamous cell carcinoma (LSCC), cyclin D1 oncogenic transformation leads to an aberrant protein expression and seems to affect the biological behaviour of the neoplasm. The aim of this study was to determine the correlation of cyclin D1 numerical imbalances with the corresponding protein expression levels in LSCCs. Material and Method: Using tissue microarray (TMA) technology, fifty $(\mathrm{n}=50)$ histologically confirmed primary LSSCs were cored at a diameter of $1.5 \mathrm{~mm}$. Immunohistochemistry (IHC) and chromogenic in situ hybridization (CISH) analyses were applied. Concerning the screening process in CISH slides, a novel real-time reference and calibration grid platform was implemented. Results: Protein overexpression was observed in 22/50 (44\%) cases; whereas, gene amplification was seen in 13/50 (26\%) cases ( $p=0.02)$. Combined protein/ gene deregulation was associated with the stage of malignancy $(p=0.0014, p=0.001)$, whereas overall protein expression was strongly correlated with the grade of tumour $(p=0.001)$. Conclusion: Cyclin D1 gene amplification led to aberrant protein expression in LSCCs and it was also correlated with an aggressive biological behaviour. To best of our knowledge, this study was the first described grid based CISH analysis under conventional bright field microscopy for detecting gene numerical imbalances while providing a novel and accurate description for screening-mapping process in the entire slide area.
\end{abstract}

Keywords: Larynx- carcinoma- cyclin- protein- gene- grid

Asian Pac J Cancer Prev, 21 (2), 379-384

\section{Introduction}

Laryngeal squamous cell carcinoma (LSCC) is the most common malignancy in larynx anatomical region. Main pathogenic factors associated with LSCC are tobacco and alcohol chronic consumption. Additionally, persistent viral (Human Papilloma Virus -HPV) infection is responsible for malignant transformation of the corresponding laryngeal epithelia (Mangano, et al., 2015). Gross Chromosome Instability (CI- polysomy/ aneuploidy) and specific gene alterations (amplification, deletion, point mutations or epigenetic: aberrant promoter methylation) are critical genomic imbalances implicated in the development and progression of LSCC, as observed in solid malignancies with different origins (Grade et al., 2015; Polyak et al., 2009). In fact, these genetic events are observed even in hyperplastic and dysplastic laryngeal epithelia as early as genetic events. Among the genes involved crucially in the carcinogenetic process, cyclins represent important markers due to their influence on the cell cycle progression and stability (Bergshoeff et al., 2014). Activation of cyclins directly stimulates the progression of cell cycle and indirectly enhances cell proliferation via interactions with specific catalytic cyclin-dependent kinases (cdks). Members of cyclin D sub-family induce cell cycle progression by inducing retinoblastoma $(\mathrm{Rb})$ protein phosphorylation. Especially, cyclin D1 (CCND1 gene location: 11q13) acts at the middle of G1 phase by activating cdk4 (Almadori et al., 2002). Cyclin D1 overexpression is detected frequently in a variety of epithelial malignancies (Aboushousha et al., 2018; Tsiambas et al., 2007; Sawair et at., 2016). In the current retrospective experimental study, we co-analyzed cyclin D1 numerical imbalances and the corresponding

${ }^{1}$ Ist ENT Department, Hippocration Hospital, Medical School, University of Athens, ${ }^{2}$ Department of Pathology-Cytology, 401 GAH, ${ }^{3}$ Department of Pathology, 417 VA Hospital (NIMTS), 7Theoretical and Physical Chemistry Institute, Photonics for Nanoapplications Laboratory, National Hellenic Research Foundation, Athens, ${ }^{4}$ Department of Physiology, ${ }^{5}$ Department of Maxillofacial, Medical School, University of Ioannina, ${ }^{6}$ Department of Otorhinolaryngology, Head and Neck Surgery, Medical School, University of Patras, Greece. *For Correspondence: tsiambasecyto@yahoo.gr. Efthymios Kyrodimos and Evangelos Tsiambas and Christos Riziotis have equal contribution in this study. 
protein expression patterns in LSCC tissues. To best of our knowledge, this was the first study that described grid-based CISH analysis under conventional bright field microscopy for detecting gene numerical imbalances while providing a novel and accurate description for screening-mapping process in the entire slide area.

\section{Materials and Methods}

\section{Patients and tissue specimens}

To perform this study, we obtained fifty $(n=50)$ formalinfixed and paraffin-embedded tissue samples of histologically confirmed primary LSCCs from patients who had undergone laryngectomy or hemilaryngectomy (including gross core biopsies in non- surgically resected cases) between 2007 and 2011 at the departments of Otorhinolaryngology- Head and Neck Surgery of the "Hippokrateion" and 417 Veterans Administration Hospital (NIMTS), Athens, Greece. All the patients were used to smoking. There were forty-five male patients with a median age of 61.5 years old and five female patients with a median age of 66.3 years old. For the control group, five specimens with histologically benign epithelia under conventional microscopy were also used. Given that no patients had a familial history of LSSC or inheritable cancer syndromes, the carcinomas were characterized as sporadic. Additionally, none of them had a history or positive DNA analysis for HPV infection. The local ethical committee consented to the use of these tissues (within the pathology department of the 417 Veterans Administration Hospital) for research purposes according to World Medical Association Declaration of Helsinki guidelines (2008). The tissue samples were fixed in 10 per cent neutral-buffered formalin. Haematoxylin and eosin $(\mathrm{H}$ and $\mathrm{E})$ stained slides of the corresponding samples were reviewed to confirm histopathological diagnosis. All lesions were graded and staged according to the histological classification criteria presented by World Health Organization (WHO) and TNM staging system for head and neck cancers (Barnes et al., 2005). Clinicopathological data of the samples are demonstrated in Table 1 .

\section{Tissue microarray (TMA) construction}

Areas of interest were identified in H\&E stained slides by a conventional microscope (Olympus BX-50, Melville, NY, USA). Selection of those areas was performed on the basis of tumour sufficiency while avoiding sites of necrosis or bleeding. Having used ATA-100 apparatus (Chemicon International, Temecula, CA, USA), all of the source blocks were 1.5 -mm diameter tissue cylindrical cores were transferred from each conventional donor block to the recipient blocks. After 3-mm microtome sectioning and $\mathrm{H}$ and $\mathrm{E}$ staining, the final constructed TMA blocks contained cores of tissue cylindrical specimens regarding the examined lesions carcinomas and benign epithelia. We observed microscopically that all of the examined samples were represented by tissue spots, confirming the adequacy of the examined specimens.
Antibody and IHC assay

We selected and applied a specific mouse monoclonal antibody anti-cyclin D1 (clone DSC6- Dako, Glostrup, Denmark) at dilution of 1:50. IHC protocol was carried out on 3-lm-thick paraffin section of the corresponding block. Tissue microarray sections initially deparaffinized in xylene, rehydrated via graded ethanol, and microwavetreated in target retrieval solution, pH 9.9 (Dako, Glostrup, Denmark). Immunostaining process for the applied marker was based on EnVision (DAKO, Glostrup, Denmark) assay using an automated staining system (I 6000-Biogenex, CA, USA) and according to corresponding manufacturer's instructions. This specific assay is based on a soluble, dextran-polymer system, preventing endogenous biotin reaction and increasing the quality of stained slides. Briefly, the sections, after peroxidise blocking, were incubated with primary antibody for 30-60 min, depending on the corresponding antibody, at room temperature and then incubated with Horseradish peroxidase labeled polymer-HRP LP for $30 \mathrm{~min}$. The antigen-antibody reaction was visualized using 3, 3'-diaminobenzidine tetrahydrochloride (DAB) as a chromogen substrate. Finally, tissue sections were slightly counterstained with hematoxylin for 30 s, dehydrated, and mounted. For negative control slides, the primary antibody was omitted. Nuclear and perinuclear- cytoplasmic expression was acceptable for cyclin D1 protein. Staining intensity levels (score 0-3) were evaluated by two independent observers (pathologists). Conflicting observations were low $(<6 \%)$ for all made evaluations. Score 2 and 3 were considered positive for cyclin D1 overexpression (Figure 1a-b).

\section{Probe and CISH assay}

Cyclin D1 gene status was determined using the ready to use SPOT LIGHT CYCLIN D1 DNA Probe (Zymed/InVitrogen, San Fransisco, USA). This digoxygenin-labeled probe is located on 11q13 and covers the entire gene area. Two slides were rinsed in deionised water and then placed in a coplin jar containing CISH FFPE Pretreatment Buffer (CISH Tissue Pre-treatment Kit, Zymed/InVitrogen, San Fransisco, USA). For heat pretreatment, the coplin jar was capped, loosely screwed, placed in a pressure cooker, and timed for 10 min after the pressure built up. The slides, then, were immediately washed in deionised water followed by enzyme digestion, which was performed by covering the sections with pepsin (CISH Tissue Pre-treatment $\mathrm{Kit}$, Zymed) for $5 \mathrm{~min}$ at $37^{\circ} \mathrm{C}$. The slides were washed with deionised water, dehydrated with graded ethanol, and air-dried. Ready to use digoxigenin-labeled Cyclin D1 gene was applied. Twenty microliters of probe was rinsed to each of the TMA sections. The tissue sections containing the added probe were denatured by placing the slides in a polymerase chain reaction (PCR) machine equipped with a slide block at $94^{\circ} \mathrm{C}$ for $5 \mathrm{~min}$. The slides were then placed in a moist slide box and incubated at $37^{\circ} \mathrm{C}$ for overnight hybridization. The sections were stringently washed in 0.59 standard saline citrate at $75^{\circ} \mathrm{C}$ for $5 \mathrm{~min}$. The CISH Polymer and the Horseradish (HRP) Detection Kit (Zymed/InVitrogen, San Fransisco, USA)-containing similar steps to IHC-were used. Shortly 
afterwards TMA sections were placed in $3 \% \mathrm{H}_{2} \mathrm{O}_{2}$ and diluted with methanol for 10 min to block endogenous peroxidase. To block unspecific staining, Cas BlockTM (Zymed/InVitrogen, San Fransisco, USA) was applied and incubated for $10 \mathrm{~min}$. Following incubation with mouse anti-dig for $30 \mathrm{~min}$ and then polymerised HRP conjugated anti-mouse for $30 \mathrm{~min}$, the probe was visualized by DAB development (CISH Polymer Detection Kit, Zymed). TMA sections were lightly counterstained with hematoxylin and dehydrated in graded ethanol. At the end of the process, CISH gene copies were easily visualized as dark brown scattered or in small clusters dots, using a conventional, bright-field microscope (Figure 1b). Interpretation of Cyclin D1 gene signal results was based on Zymed's Evaluation Chart for CISH. According to this guide, two gene copies per nucleus demonstrated normal gene pattern; whereas, $6-10$ or small clusters characterized a low-level gene amplification. High gene amplification level was characterized by the presence of more than 10 gene copies or clusters of them per nucleus in more than $50 \%$ of the examined cells (Figure 1c). In borderline cases, a second slide should be analyzed for chromosome 13 centromere copies in order to determine pure gene amplification or polysomy. In the current CISH TMAs CISH analysis, borderline cases were not identified.

\section{Slide screening process}

Screening procedure regarding the CISH stained slides was derived under bright field microscopy (microscope Olympus CX-31, Menvile, NY, USA, with ToupView image analysis software, ProWay/ToupTek Protonics, Cn/USA) with combined $100 \mathrm{X} / 400 \mathrm{X}$ magnification. Concerning the CISH slides, screening was based on a set of cover slips integrated with spatial rectangular grid (now GCS) in order to perform a systematic way of slide eye-scanning. We used in this study a novel technique of micromachining with the use of ultra short Laser pulses at the femtosecond pulse duration regime (Femtosecond Laser Micromachining-FLM) in combination with high precision translation systems (Tsiambas and Riziotis, 2017). Laser techniques can allow direct writing and transfer of predetermined patterns by means of surface or sub-surface micromachining in a variety of materials ranging from glasses to soft polymeric materials. The technique of FLM inscription was applied here for the first time towards the fabrication of a visible rectangular grid in a microscope CISH slide's cover slip for medical diagnostic purposes.

Adjusting the laser writing characteristics the inscripted grid lines' width could be typically ranging from $5 \mu \mathrm{m}$ to $500 \mu \mathrm{m}$. We used commercially available cover slips with length of $50 \mathrm{~mm}$, width of $24 \mathrm{~mm}$, and thickness $0.5 \mathrm{~mm}$ (Menarini, IT), which were made by typical borosilicate based glass. This prototype grid consists of seventy- two $(n=72)$ rectangular square areas arranged in 12 columns and 6 rows. Each square segment has a typical surface area of $4 \mathrm{~mm} \times 4 \mathrm{~mm}$ equal to $16 \mathrm{~mm}^{2}$. Each square segment can have appropriate indexing marks printed with various techniques like laser based micromachining in a suitable way to assure minimum visual interference under microscope inspection. In the present study, the indexing was provided by sequential numbering of the square cells as indicated schematically in Figure 1d. In the specific example due to the selection of grid's cells size, appear also at the right part of the grid six residual non-rectangular cells, numbered at the figure as 73-78 that do not affect of course the generalization of the proposed grid's architecture. The grid's size and cells' density could be modified according to screener's needs but here it was selected a specific size believing to be a well balanced choice according to our experience. At the end of the technical grids' fabrication process, a new set of five $(n=5)$ GCS was constructed.

\section{Statistical analysis}

Descriptive statistics were performed for data analysis. Associations between variables including protein expression levels, gene copies numerical variants, and clinicopathological parameters such as sex, tumor grade and stage, anatomic location, and alcohol consumption were studied using Pearson Chi square test $\left(\chi^{2}\right)$ estimated along with its 99\% CI (SPSS v20 (SPSS Inc, Chicago, IL, USA). Two-tailed p-values $\leq 0.05$ were considered statistically significant. Results and correlations ( $p$-values) are described in Table 1.

\section{Results}

All of the examined cases were evaluated properly according to IHC analysis, demonstrating different cyclin D1 expression levels. Increased (Score 2 and 3) protein expression was observed in 22/50 (44\%) tissue cores regarding LSCCs. Overall cyclin D1 expression was associated to stage and grade of the examined patients $(p=0.001, p=0.0014$, respectively); whereas, no other statistical correlations were identified concerning the rest clinicopathological parameters (gender: $\mathrm{p}=0.201$, anatomic location: $\mathrm{p}=0.44$, alcohol consumption $\mathrm{p}=0.22$ ). Concerning CISH grid-based screening analysis, 13/50 (26\%) tissue cores demonstrated low to high gene amplification (isolated multiple gene copies $>=6$ and gene clusters), while the rest $37 / 50$ (64\%) were found to be normal diploid (2-3 signals per nucleus). Overall gene numerical imbalances (amplification) were associated strongly with the stage of the tumours and the history of alcohol consumption ( $p=0.001, p=0.0092)$; whereas, no other statistical correlations were identified concerning the rest clinicopathological parameters (gender: $\mathrm{p}=0.234$, anatomic location: $\mathrm{p}=0.92$, grade: $\mathrm{p}=0.74$ ). Comparing IHC and CISH results, gene amplification was correlated to protein overexpression $(\mathrm{p}=0.02)$.

\section{Discussion}

Extensive histogenetic analyses have shown that CI combined or not with specific oncogene over activation and suppressor gene inactivation is correlated with the progression of histopathologically confirmed premalignant lesions (dysplasia) to LSCC in laryngeal epithelia (Hanken et al., 2014). In fact, CI is strongly correlated with an increased risk for malignant transformation in 


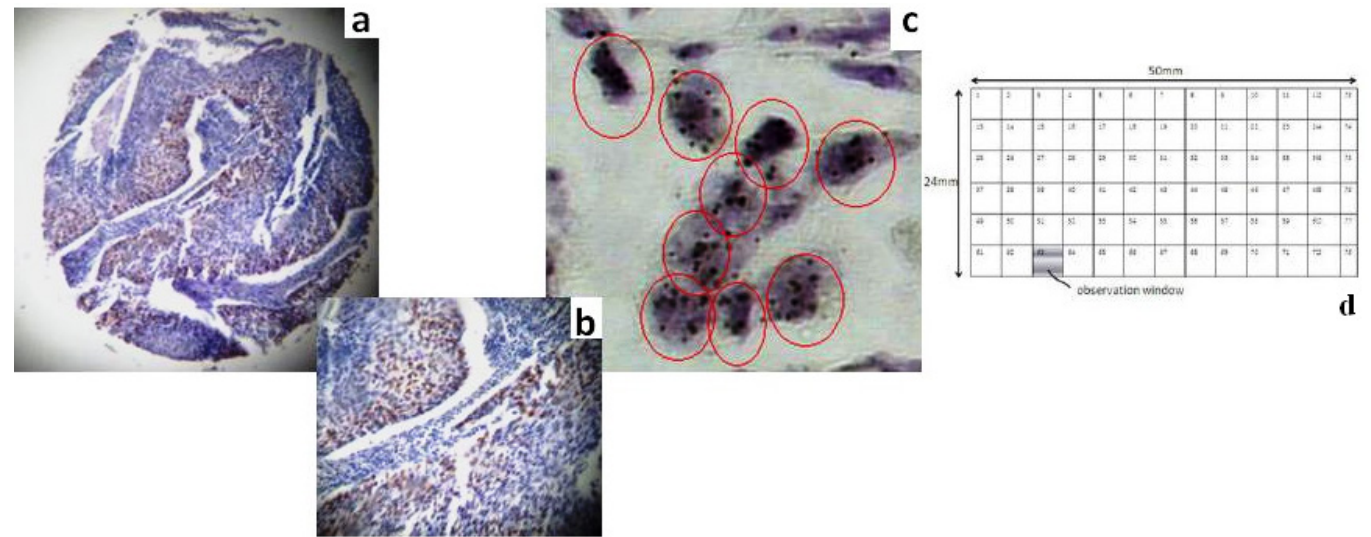

Figure 1. Cyclin D1 IHC and CISH Analyses on TMA Cylindrical Cores a. A LSCC tissue core demonstrates protein overexpression. Note a significant number of dark brown stained nuclei (IHC SCORE 3, anti-cyclin D1, DAB chromogen; original magnification40x) b. The same core is shown at magnification of 100x c. A LSCC tissue core demonstrates gene amplification. Note multiple scattered or in clusters gene signals (inside red circles DAB chromogen; original magnification 400x) d. Schematic presentation of the grid-based cover slip that was implemented in CISH slide screening-mapping. Note this prototype grid consisting of seventy- two $(n=72)$ rectangular square areas arranged in 12 columns and 6 rows.

hyperplastic and moderate to severe dysplastic epithelia. Focusing on cyclin D1 (CCND1 gene band) amplification, based on Fluoresence in situ hybridization (FISH) and Array GeneChip analyses several studies observed that significant proportions of protein overexpressed cases demonstrate this molecular specific mechanism of gene deregulation. Interestingly, the highest rates of cyclin D1 gene amplification are observed in Head and Neck Squamous Cell Carcinoma (HNSCC), especially regarding not LSCC, but also pharyngeal and Oral Squamous Cell Carcinomas (OSCCs) (Niméus et al.,
2014).

In the current study, we applied a combined IHC and CISH protocol in order to explore the role of protein and gene cyclin D1 deregulation in LSCC TMAs. Although CISH molecular method is a fine alternative to FISH for analyzing tissues or TMAs in solid malignancies, there are very limited data on LSCC, in contrast to other malignancies such as breast carcinoma (Sui et al., 2014; Wang et al., 2014; Lundgren et al., 2012). Our research study showed that simultaneous protein and gene deregulation was associated with the stage of malignancy;

Table 1. Clinicopathological Parameters and Total Cyclin D1 IHC \& CISH Results

\begin{tabular}{|c|c|c|c|c|c|c|c|}
\hline \multicolumn{2}{|c|}{ Clinicopathological parameters } & \multicolumn{2}{|c|}{$\mathrm{IHC}$} & \multirow[t]{2}{*}{ P-value } & \multicolumn{2}{|c|}{$\mathrm{CISH}$} & \multirow[t]{2}{*}{ P-value } \\
\hline & $(\mathrm{n}=50)$ & $\mathrm{OE}$ & LE & & Amplified & Non Amplified & \\
\hline LSCC & & $22 / 50(44 \%)$ & $28 / 50(56 \%)$ & & $13 / 50(26 \%)$ & $37 / 50(74 \%)$ & \\
\hline Gender & & & & 0.201 & & & 0.234 \\
\hline Male & $45(90 \%)$ & $21 / 50$ & $24 / 50$ & & $12 / 50$ & $33 / 50$ & \\
\hline Female & $5(10 \%)$ & $1 / 50$ & $4 / 50$ & & $1 / 50$ & $4 / 50$ & \\
\hline Anatomical location & & & & 0.44 & & & 0.92 \\
\hline Supraglotis & $5(10 \%)$ & $2 / 50$ & $3 / 50$ & & $1 / 50$ & $4 / 50$ & \\
\hline Transglotis & $45(64 \%)$ & $20 / 50$ & $25 / 50$ & & $12 / 50$ & $33 / 50$ & \\
\hline Grade & & & & 0.001 & & & 0.74 \\
\hline 1 & $4(8 \%)$ & $2 / 50$ & $2 / 50$ & & $0 / 50$ & $4 / 50$ & \\
\hline 2 & $19(38 \%)$ & $10 / 50$ & $9 / 50$ & & $5 / 50$ & $14 / 50$ & \\
\hline 3 & $27(54 \%)$ & $10 / 50$ & $17 / 50$ & & $8 / 50$ & $19 / 50$ & \\
\hline Stage & & & & 0.0014 & & & 0.001 \\
\hline I & $0(0 \%)$ & $0 / 50$ & $0 / 50$ & & $0 / 50$ & $0 / 50$ & \\
\hline II & $4(8 \%)$ & $2 / 50$ & $2 / 50$ & & $0 / 50$ & $4 / 50$ & \\
\hline III & $25(50 \%)$ & $5 / 50$ & $20 / 50$ & & $1 / 50$ & $24 / 50$ & \\
\hline IV & $21(42 \%)$ & $15 / 50$ & $6 / 50$ & & $12 / 50$ & $9 / 50$ & \\
\hline Alcohol consumption & & & & 0.22 & & & 0.009 \\
\hline Yes & $43(84 \%)$ & $19 / 50$ & $24 / 50$ & & $10 / 50$ & $33 / 50$ & \\
\hline No & $7(16 \%)$ & $3 / 50$ & $4 / 50$ & & $3 / 50$ & $4 / 50$ & \\
\hline
\end{tabular}

LSCC, Laryngeal Squamous Cell Carcinoma; IHC, Immunohistochemistry; OE, Over expression; LE, Low expression; CISH, Chromogenic in situ hybridization. 
whereas, overall protein expression was strongly correlated with the grade of tumour. Additionally, gene amplification seems to be a significant genetic mechanism leading to protein overexpression in these malignancies. Concerning the current molecular results, another study observed that although a significant proportion of examined LSCCs demonstrated cyclin D1 gene amplification, the main anatomic location in HNSCCs characterized by this mechanism was the hypo-pharyngeal epithelia, an indirect evidence of a site-specific prevalence for oncogene amplifications (Blessmann et al., 2013). Furthermore, another study co-analyzed CCND1 numerical imbalances and a specific $\mathrm{A} / \mathrm{G}$ polymorphism in exon 4 based on differential and RFLP- Polymerase chain reaction (PCR) assays, respectively. They reported D1 A870G polymorphism and increased susceptibility for LSCC development at the glottic region (Verim et al., 2013). Similarly, another study reported carriers of cyclin D1 G870A and G1722C polymorphisms, especially among smokers, were characterized by increased risk for developing HNSCC (Lin et al., 2014), which is another paradigm of a site-specific prevalence regarding cyclin D1 oncogene transformation. Based on these studies, it seems that gene amplification, although significant,- is not the only cyclin D1 deregulation mechanism. Finally, the current experimental study was characterized with the pilot implementation of an innovative reference and calibration grid on conventional cover slips in the screening process of CISH slides. We already reported this improved technique as a tool for systematic screening in immunocytochemistry stained slides (Tsiambas et al., 2018).

In conclusion, our combined IHC and CISH analysis on LSCC TMAs showed that cyclin D1 gene amplification was a strong deregulation mechanism leading to aberrant over-expression of the corresponding protein product. In addition, it was correlated with aggressive phenotype in a subset of them. Controversial published results regarding cyclin D1 gene expression patterns in LSCC should be explained at the basis of selecting patients with different clinico-histogenetic characteristics. In conjunction to this, grid-based CISH analysis, under conventional bright field microscopy, is an easy to use molecular method for specific detecting genetic signatures in LSCC patients based on gene numerical imbalances.

\section{Acknowledgements}

This work was supported in part by the project “Advanced Materials and Devices" (MIS 5002409), which is implemented under the "Action for the Strategic Development on the Research and Technological Sector", funded by the Operational Programme "Competitiveness, Entrepreneurship and Innovation" (NSRF 2014-2020) and co-financed by Greece and the European Union (European Regional Development Fund). COST Action BM1401 "Raman based applications for clinical diagnostics- Raman4Clinics", and COST Action MP1401: "Advanced fibre laser and coherent source as tools for society, manufacturing and lifescience" are also acknowledged. Authors acknowledge the significant scientific contribution of George Vilaras, Technologist in Dept of Pathology, Medical School, University of Athens, Athens, Greece as an expert in IHC/ICC/CISH techniques.

\section{Conflicts of interest}

The authors declare no conflicts of interest.

\section{References}

Aboushousha T, Hammam O, Helal N, et al (2018). Impact of Cyclin D1 and heterogeneous nuclear ribonucleoprotein-K (HnRNP-K) on urinary bladder carcinogenesis. Asian Pac J Cancer Prev, 19, 513-9.

Almadori G, Galli J, Cadoni G, et al (2002). Human papillomavirus infection and cyclin D1 gene amplification in laryngeal squamous cellcarcinoma: biologic function and clinical significance. Head Neck, 24, 597-604.

Barnes L, Eveson JW, Reichart P, et al (2005) Pathology and Genetics: Head and Neck Tumours. WHO IARC Press, Lyon, Fr, pp 118-30.

Bergshoeff VE, Van der Heijden SJ, Haesevoets A, et al (2014). Chromosome instability predicts progression of premalignant lesions of the larynx. Pathology, 46, 216-24.

Blessmann M, Al-Dam A, Hanken H, et al (2013). Amplification of the PPFIA1 gene region on 11q13 in oral squamous cell carcinomas (OSCC). J Craniomaxillofac Surg, 41, 845-9.

Grade M, Difilippantonio MJ, Camps J (2015). Patterns of chromosomal aberrations in solid tumors. Recent Results Cancer Res, 200, 135-40.

Hanken H, Gröbe A, Cachovan G, et al (2014). CCND1 amplification and cyclin D1 immunohistochemical expression in head and neck squamous cell carcinomas. Clin Oral Investig, 18, 269-76.

Lin H, Fang L, Lin D (2014). Association of cyclin D1 variants with head and neck cancer susceptibility: evidence from a meta-analysis. Asian Pac J Cancer Prev, 15, 5645-51.

Lundgren K, Brown M, Pineda S, et al (2012). Effects of cyclin D1 gene amplification and protein expression on time to recurrence in postmenopausal breast cancer patients treated with anastrozole or tamoxifen: a TransATAC study. Breast Cancer Res, 14, 2-1.

Mangano A, Mangano A, Lianos GD, et al (2015). Head and neck squamous cell carcinoma and human papillomavirus: epidemiology, treatment and future trends. Future Oncol, 11, 889-91.

Niméus E, Baldetorp B, Bendahl PO, et al (2014). Amplification of the cyclin D1 gene is associated with tumour subsite, DNA non-diploidy and high S-phase fraction in squamous cell carcinoma of the head and neck. Oral Oncol, 40, 624-9.

Polyak K, Haviv I, Campbell IG (2009). Co-evolution of tumor cells and their microenvironment. Trends Genet, 25, 30-8.

Sawair F, Hassona Y, Irwin C, et al (2016). p53, Cyclin D1, p21 (WAF1) and Ki-67 (MIB1) expression at invasive tumour fronts of oral squamous cell carcinomas and development of local recurrence. Asian Pac J Cancer Prev, 17, 1243-9.

Sui JQ, Xie KP, Zou W, et al (2014). Emodin inhibits breast cancer cell proliferation through the ER $\alpha$-MAPK/Akt-cyclin D1/Bcl-2 signaling pathway. Asian Pac J Cancer Prev, 15, 6247-51.

Tsiambas E, Karameris A, Gourgiotis S, et al (2007). Simultaneous deregulation of $\mathrm{p} 16$ and cyclin D1 genes in pancreatic ductal adenocarcinoma: a combined immunohistochemistry and image analysis study based on tissue microarrays. JBUON, 12, 261-7.

Tsiambas E, Riziotis C (2017). Implementation of a real-time reference and calibration grid platform for improved 
screening - mapping in Pap test slides. Pathol Int, 67, 24-31.

Tsiambas E, Riziotis C, Mastronikolis NS, et al (2018). Comparative p16IKN4A expression in laryngeal carcinoma and cervical cancer precursors: A real-time grid-based immunocytochemistry analysis. Anticancer Res, 38, 5805-10.

Verim A, Ozkan N, Turan S, et al (2013). Association of the Cylin D1 G870A polymorphism with laryngeal cancer: are they really related?. Asian Pac J Cancer Prev, 14, 7629-34.

Wang CD, Yuan CF, Bu YQ, et al (2014). Fangchinoline inhibits cell proliferation via Akt/GSK-3beta/ cyclin D1 signaling and induces apoptosis in MDA-MB-231 breast cancer cells. Asian Pac J Cancer Prev, 15, 769-73.

\section{๑๐。}

This work is licensed under a Creative Commons AttributionNon Commercial 4.0 International License. 